\title{
Development of RD50-MPW2: a high-speed monolithic HV-CMOS prototype chip within the CERN-RD50 collaboration
}

\author{
Chenfan Zhang ${ }^{1, a, b}$, Gianluigi Casse ${ }^{a, b}$, Nicola Massari ${ }^{b}$, Eva Vilella ${ }^{a}$, Joost \\ Vossebeld $^{\text {a }}$ \\ aDepartment of Physics, University of Liverpool \\ Oliver Lodge Laboratory, Oxford Street, L69 7ZE Liverpool, United Kingdom \\ ${ }^{b}$ IRIS, Fondazione Bruno Kessler (FBK) \\ Via Sommarive, 18, Povo, 38123, Trento, Italy \\ E-mail: chenfan@hep.ph.Iiv.ac.uk
}

The CERN-RD50 collaboration has ongoing research to further develop monolithic High VoltageCMOS (HV-CMOS) sensors in a $150 \mathrm{~nm}$ process for future particle physics experiments. As a part of this research programme, a test chip (RD50-MPW2) that implements new methodologies for low leakage current and fast and low-noise readout circuitry has been designed and submitted for fabrication. This article presents the design details and simulation results of the $8 \times 8$ matrix of high-speed monolithic HV-CMOS pixels included in RD50-MPW2, in which two flavours of fast pixels are implemented: a conventional continuous-reset pixel and a switched-reset pixel with a novel asynchronous switched-reset scheme.

TWEPP 2019 Topical Workshop on Electronics for Particle Physics

2-6 September 2019

Facultad de Quimica de la Unicersidad de Santiago de Compostela, Santiago de Compostela, Spain

\section{${ }^{1}$ Speaker}




\section{Introduction}

Because of their excellent radiation tolerance to high integrated fluences $\left(2 \times 10^{16} 1 \mathrm{MeV}\right.$ $\left.\mathrm{n}_{\mathrm{eq}} / \mathrm{cm}^{2}\right)$, precise time resolution $(\sim 1 \mathrm{~ns})$ and capability to cope with high hit rates $\left(\sim 3 \mathrm{GHz} / \mathrm{cm}^{2}\right)$, hybrid pixel detectors are widely used in current particle physics experiments [1]. However, due to their multi-layer nature, hybrid detectors have substantial thickness $(300 \mu \mathrm{m})$, which limits their accuracy in particle tracking. In addition, the bump-bonding process to assemble the sensor and the readout chip is expensive $\left(\sim 1 \mathrm{M} £ / \mathrm{m}^{2}\right)$, time-consuming and restricts pixels to minimum sizes of $55 \mu \mathrm{m} \times 55 \mu \mathrm{m}$, making it challenging to achieve fine granularity.

Alternatives to the hybrid detectors are the monolithic sensors in commercial CMOS processes [2]. Since the sensing diode and the readout electronics are integrated into a single layer of silicon, CMOS sensors avoid the complex hybridization process. The one-layer structure allows thinning the detectors down to $50 \mu \mathrm{m}$. However, their slow charge collection by diffusion and low radiation tolerance $\left(10^{13} 1 \mathrm{MeV} \mathrm{n} \mathrm{n}_{\mathrm{eq}} / \mathrm{cm}^{2}\right)$ [3] limit the application of CMOS sensors to low rate and low radiation experiments only, such as the ALICE Inner Tracker System (ITS) Upgrade. To achieve monolithic sensors with advanced performance, High Voltage CMOS (HV-CMOS) sensors [4] are being developed for faster charge collection by drift and higher radiation tolerance $\left(10^{15} 1 \mathrm{MeV} \mathrm{n}_{\mathrm{eq}} / \mathrm{cm}^{2}\right)$ [5]. The Mu3e experiment at the Paul Scherrer Institute (PSI) in Switzerland has adopted monolithic HV-CMOS sensors for its pixel tracker [6] and other experiments, such as the LHCb Upgrade Ib and II and CLIC, are considering this sensor technology as well.

This article describes ongoing research within the CERN-RD50 collaboration to further develop monolithic HV-CMOS sensors in a $150 \mathrm{~nm}$ process for future particle physics experiments. In particular, it focuses on the design work of RD50-MPW2, the second prototype developed by the collaboration, aimed at minimizing the leakage current of the sensor and developing fast and low-noise readout circuitry.

\section{RD50-MPW2}

The layout of RD50-MPW2 is shown in figure 1(a). The chip is produced on $10 \Omega \cdot \mathrm{cm}, 200$ - $500 \Omega \cdot \mathrm{cm}, 1.9 \mathrm{k} \Omega \cdot \mathrm{cm}$, and $3 \mathrm{k} \Omega \cdot \mathrm{cm}$ substrate resistivity wafers. This prototype includes test structures (marked as 1 and 6 in the figure), an $8 \times 8$ pixel matrix (2), an analog buffer (3), a Single Event Upset (SEU) tolerant memory array (4) and a bandgap voltage reference (5). Compared with the previous version RD50-MPW1, this chip reduces the leakage current by preventing certain filling layers added by the foundry and adding a series of guard rings [7].

(a)

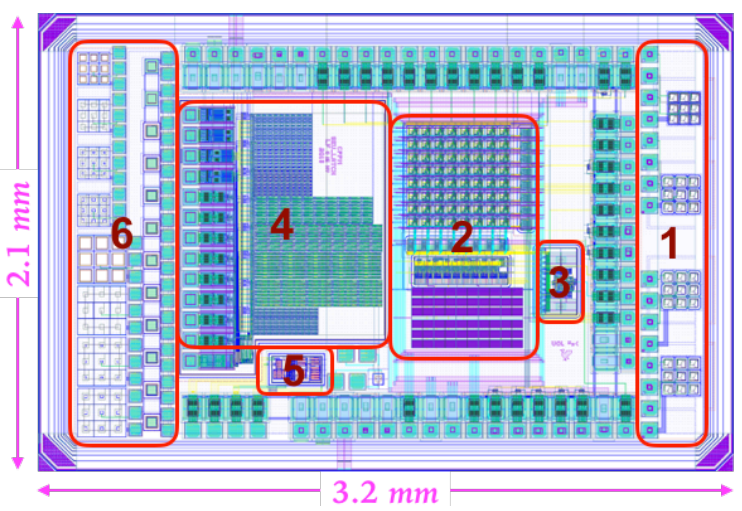

(b)

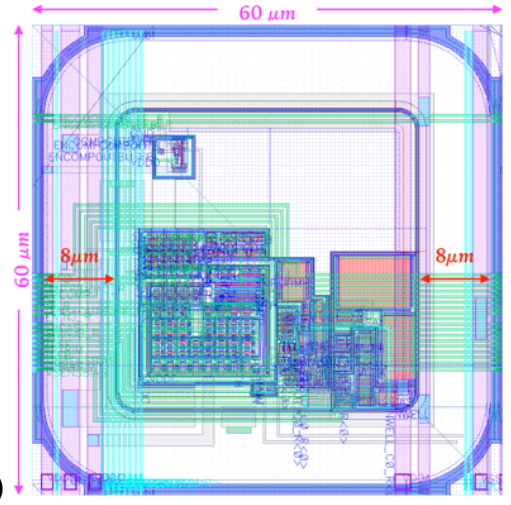

Figure 1. (a) Layout of RD50-MPW2 and (b) layout of one pixel. 


\subsection{Pixel matrix}

This article focuses on the design of the $8 \times 8$ pixel matrix, in which two flavours of fast readout circuits are implemented to verify the functionality of HV-CMOS pixels with new methodologies for low leakage current, high response rate and fine time resolution. The pixel size is $60 \mu \mathrm{m} \times 60 \mu \mathrm{m}$ as shown in the pixel layout in figure 1(b). Each pixel flavour consists of a high impedance circuit to bias the collecting electrode, a Charge Sensitive Amplifier (CSA) based on a single-ended folded cascode amplifier with PMOS input as shown in figure 2(a), a source follower, a high-pass filter and a CMOS comparator with a local 4-bit Digital to Analog Convertor (DAC) for threshold tuning. As observed, the area in the upper half of the pixel layout is empty and will be occupied by digital readout circuits in a future version of the design. Analog signals generated by the pixels at the output of the CSA and the comparator can be measured either via the analog buffer or directly through output pads.

\subsection{High response rate}

To cope with the high hit rate foreseen in future experiments $\left(2-4 \mathrm{GHz} / \mathrm{cm}^{2}\right.$ for High Luminosity-LHC), it is necessary to improve the response rate to avoid pile-ups in single pixels. A high response rate can be achieved with an amplifier that has short rising and falling edges when in response to a particle hit.

The rising edge duration is given by $t_{r i s e} \propto C_{d} / g_{m}$, where $C_{d}$ is the detector capacitance and $g_{m}$ the transconductance of the CSA input transistor (M0 in figure 2(a)). $C_{d}$ is mainly the capacitance between the P-substrate and the deep n-well ( $\mathrm{C}_{\text {sub/DN }}$ in figure 2(b)) and that between the deep p-well (PSUB) and the deep n-well (C $\left.\mathrm{C}_{\mathrm{PSUB} / \mathrm{DN}}\right)$. With a given pixel size and $\mathrm{p}$-well/deep n-well spacing, the deep n-well area and therefore $C_{\text {sub/DN }}$ are fixed. $C_{P S U B / D N}$ can be minimised by reducing the size of the PSUB layer which isolates the n-well from the deep n-well layer and thus allows full CMOS logic in the sensing area of the pixel. The values of $\mathrm{C}_{\text {sub/DN }}$ and $\mathrm{C}_{\mathrm{PSUB} / \mathrm{DN}}$, simulated with Cadence at $-60 \mathrm{~V}$ of sensor biasing, are $80 \mathrm{fF}$ and $70 \mathrm{fF}$ respectively. $g_{m}$ is increased by optimizing the geometry and the drain current of M0. With a low $C_{d}$ and a large $g_{m}$, the rising edge duration is decreased.

(a)

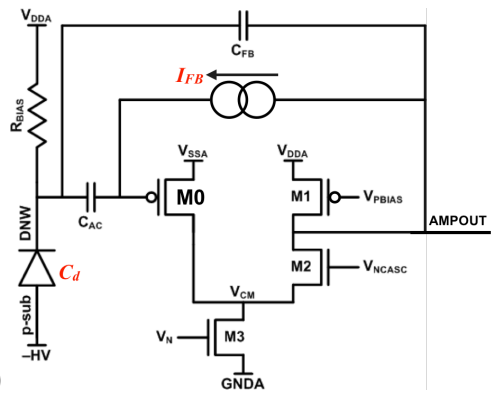

(b)

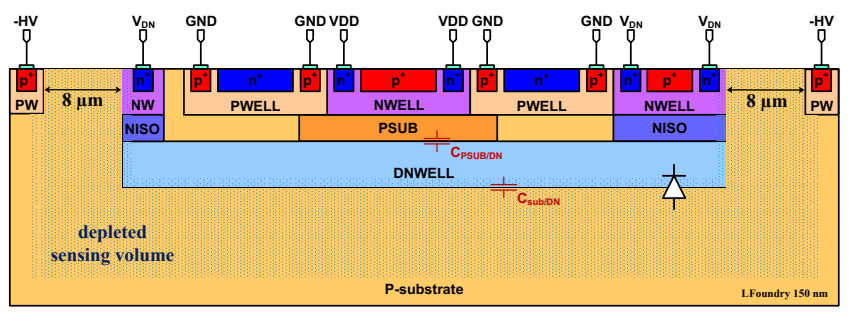

Figure 2. (a) Schematic of the CSA and (b) Cross-section of the pixel

The falling edge duration is given by $t_{\text {fall }} \propto 1 / I_{F B}$, where $I_{F B}$ is the feedback current that resets the pixel after each event. Two pixel flavours with different methods to reset the pixel are implemented in this work. One flavour is the continuous-reset pixel, which is shown in figure 3(a). In this conventional pixel flavour, its feedback current $I_{F B_{-} C O N T}$ is constant. The input charge $Q$ accumulated over the feedback capacitance is linearly discharged and the fall time can be written as $t_{\text {fall }}=Q / I_{F B_{-} \text {CONT. }}$. Thus, the time to reset the CSA is proportional to the input charge and the Time over Threshold (ToT) can be measured. Although a large $I_{F B_{-} C O N T}$ decreases $t_{\text {fall }}$, it 
also lowers the charge-to-voltage gain and increases the noise. Therefore, the falling edge duration can only be shortened to a limited extent in this pixel flavour.

The other flavour is the switched-reset pixel, shown in figure 3(b), where a switched large current $I_{F B_{-} S W}$ discharges $Q$ in less than $5 \mathrm{~ns}$. Switched-reset pixels have been explored in previous works, where a clock signal controls the switch for a synchronous periodic reset [8]. However, that option limits the reset frequency (1 MHz in [8]) and induces crosstalk noise by clock feedthrough. In this work, the switch is asynchronously controlled by the in-pixel comparator. The mechanism is as follows: 1) The output of the comparator COMPOUT toggles to high when a charged particle hits the pixel. 2) After a delay of $15 \mathrm{~ns}$, the switch is closed and $I_{F B_{-} S W}$ starts discharging $\mathrm{C}_{\mathrm{FB}}$ and resets the CSA in less than $5 \mathrm{~ns}$. 3) COMPOUT returns to its low level and opens the switch again. Because $I_{F B_{-} S W}$ flows only in the reset phase, its value can be rather high without affecting the charge sensitivity and the noise. The Time of Arrival (ToA) can be achieved. This pixel flavour is suitable for experiments that require binary information.

(a)

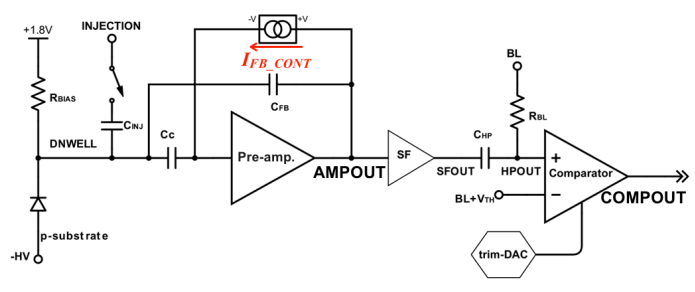

(b)

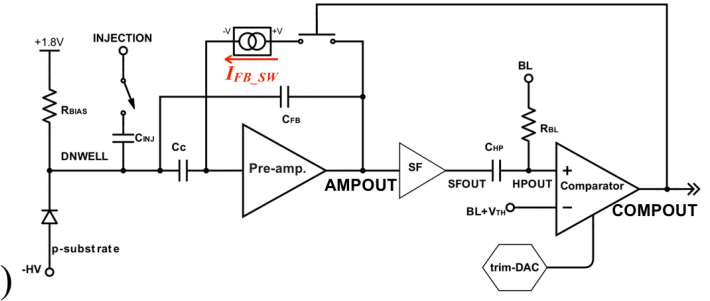

Figure 3. Schematics of (a) the continuous-reset pixel and (b) the switched-reset pixel

\subsection{Fine time resolution}

The time resolution reflects the accuracy of the ToA measurement. The ToA is recorded when the amplified signal of a particle hit surpasses the threshold voltage $\mathrm{V}_{\mathrm{TH}}$. The measured ToA depends on the charge collection time of the sensor and the response time of the front-end electronics. The uncertainties associated to these two factors cause the amplified signals of particles that generate different amounts of charges in the sensor to cross $\mathrm{V}_{\mathrm{TH}}$ with different time delays. This time difference is called time-walk and limits the time resolution.

The response time of state-of-the-art HV-CMOS sensors dominates the time-walk. Assuming the rising edges of amplified signals of two particles have slew rates of SR $-\Delta \mathrm{SR}$ and $\mathrm{SR}+\Delta \mathrm{SR}$, and the time they need to reach $\mathrm{V}_{\mathrm{TH}}$ is $\frac{\mathrm{V}_{\mathrm{TH}}}{\mathrm{SR}-\Delta \mathrm{SR}}$ and $\frac{\mathrm{V}_{\mathrm{TH}}}{\mathrm{SR}+\Delta \mathrm{SR}}$, the time difference is $\frac{\mathrm{V}_{\mathrm{TH}}}{\mathrm{SR}-\Delta \mathrm{SR}}-\frac{\mathrm{V}_{\mathrm{TH}}}{\mathrm{SR}+\Delta \mathrm{SR}}=\mathrm{V}_{\mathrm{TH}} \frac{2 \Delta \mathrm{SR}}{\mathrm{SR}^{2}-\Delta \mathrm{SR}^{2}} \approx \mathrm{V}_{\mathrm{TH}} \frac{2 \Delta \mathrm{SR}}{\mathrm{SR}^{2}}$. This expression shows that the time-walk can be reduced by decreasing $\mathrm{V}_{\mathrm{TH}}$ and increasing $\mathrm{SR}$. With the threshold voltage constrained by the noise of the sensor, the slew rate is increased by shortening the rising edge of the CSA in this work. As a result, the time resolution is improved.

\subsection{Simulation results}

Post-layout simulation results in figure 4 show the response of the continuous-reset and switched-reset pixels to input charges ranging from $1 \mathrm{ke}^{-}$to $10 \mathrm{ke}^{-}$when $I_{F B_{-} C O N T}$ and $I_{F B_{-} S W}$ are set to $15 \mathrm{nA}$ and $1 \mu \mathrm{A}$. Table 1 summarizes and compares performance of both pixel flavours together with that of the pixel in RD50-MPW1, when the input charge is $5 \mathrm{ke}^{-}$. The response time is the time the output of a hit pixel takes to recover its baseline voltage. 

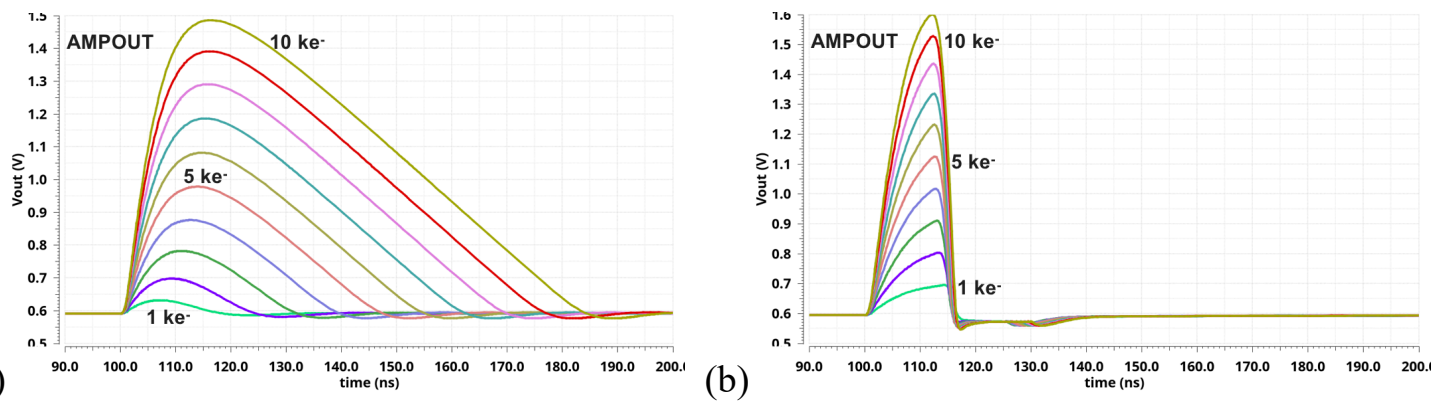

Figure 4. Simulation results of (a) the continuous-reset pixel and (b) the switched-reset pixel

\begin{tabular}{c|c|c|c|c|c|c}
\hline Input: $5 \mathrm{ke}^{-}$ & pixel size & gain & response time & ENC & time-walk & power cons. \\
\hline Continuous-reset pixel & $60 \mu \mathrm{m} \times 60 \mu \mathrm{m}$ & $50 \mu \mathrm{V} / \mathrm{e}^{-}$ & $53 \mathrm{~ns}$ & $120 \mathrm{e}^{-}$ & $7.8 \mathrm{~ns}$ & $24 \mu \mathrm{W} /$ pixel \\
\hline Switched-reset pixel & $60 \mu \mathrm{m} \times 60 \mu \mathrm{m}$ & $80 \mu \mathrm{V} / \mathrm{e}^{-}$ & $32 \mathrm{~ns}$ & $100 \mathrm{e}^{-}$ & $6.4 \mathrm{~ns}$ & $25 \mu \mathrm{W} /$ pixel \\
\hline RD50-MPW1 pixel & $50 \mu \mathrm{m} \times 50 \mu \mathrm{m}$ & $30 \mu \mathrm{V} / \mathrm{e}^{-}$ & $125 \mathrm{~ns}$ & $115 \mathrm{e}^{-}$ & $11 \mathrm{~ns}$ & $23 \mu \mathrm{W} /$ pixel \\
\hline
\end{tabular}

Table 1. Pixel performances of RD50-MPW2 and RD50-MPW1

\section{Conclusion}

The RD50-MPW2 prototype developed in the CERN-RD50 collaboration is designed to have low leakage current and fast readout circuitry. An $8 \times 8$ pixel matrix with two pixel flavours for high response rate and fine time resolution has been implemented in this chip. A novel asynchronous switched-reset front-end is proposed. Post-layout simulations of the two pixel flavours show their good performance in terms of response time (30 ns and $50 \mathrm{~ns}$ for a $5 \mathrm{ke}^{-}$ signal), time walk ( $\sim 8 \mathrm{~ns})$ and noise $\left(\mathrm{ENC} \sim 100 \mathrm{e}^{-}\right)$.

\section{Acknowledgement}

This work has been partly performed in the framework of the CERN-RD50 collaboration.

\section{References}

[1] M. Garcia-Sciveres, N. Wermes, A review of advances in pixel detectors for experiments with high rate and radiation, Reports on Progress in Physics, v. 81, n. 6, 2018.

[2] R. Turchetta et al, A monolithic active pixel sensor for charged particle tracking and imaging using standard VLSI CMOS technology, Nucl. Instr. and Meth. A 458 (2001) 677-689.

[3] M. Mager, ALPIDE, the Monolithic Active Pixel Sensor for the ALICE ITS upgrade, Nucl. Instr. and Meth. A 824 (2016) 434-438.

[4] I. Peric, A novel monolithic pixelated particle detector implemented in high-voltage CMOS technology, Nucl. Instr. and Meth. A 582 (2007) 876-885.

[5] M. Barbero, Radiation hard DMAPS pixel sensors in $150 \mathrm{~nm}$ CMOS technology for operation at LHC, arXiv:1911.01119v1 [physics.ins-det].

[6] A. Blondel et al., Research proposal for an Experiment to Search for the Decay $\mu \rightarrow e e e$, arXiv:1301.6113v1.

[7] M. Franks, Design optimisation of depleted CMOS detectors using TCAD simulations within the CERN-RD50 collaboration, URL: https://indico.cern.ch/event/777112/contributions/3314468/

[8] M. Havránek, Development of pixel front-end electronics using advanced deep submicron CMOS technologies, $\mathrm{PhD}$ thesis, (2014). URL: http://cds.cern.ch/record/2317324 